\title{
The construction and validation of an RNA binding protein-related prognostic model for bladder cancer
}

Fengxia Chen ${ }^{1,2}$, Qingqing Wang ${ }^{1,2}$ and Yunfeng Zhou ${ }^{1,2^{*}}$

\begin{abstract}
Background: RNA-binding proteins (RBPs) play crucial and multifaceted roles in post-transcriptional regulation. While RBPs dysregulation is involved in tumorigenesis and progression, little is known about the role of RBPs in bladder cancer (BLCA) prognosis. This study aimed to establish a prognostic model based on the prognosis-related RBPS to predict the survival of BLCA patients.

Methods: We downloaded BLCA RNA sequence data from The Cancer Genome Atlas (TCGA) database and identified RBPs differentially expressed between tumour and normal tissues. Then, functional enrichment analysis of these differentially expressed RBPs was conducted. Independent prognosis-associated RBPs were identified by univariable and multivariable Cox regression analyses to construct a risk score model. Subsequently, Kaplan-Meier and receiver operating characteristic curves were plotted to assess the performance of this prognostic model. Finally, a nomogram was established followed by the validation of its prognostic value and expression of the hub RBPs.

Results: The 385 differentially expressed RBPs were identified included 218 and 167 upregulated and downregulated RBPs, respectively. The eight independent prognosis-associated RBPS (EFTUD2, GEMIN7, OAS1, APOBEC 3H, TRIM71, DARS2, YTHDC1, and RBMS3) were then used to construct a prognostic prediction model. An indepth analysis showed lower overall survival (OS) in patients in the high-risk subgroup compared to that in patients in the low-risk subgroup according to the prognostic model. The area under the curve of the time-dependent receiver operator characteristic (ROC) curve were 0.795 and 0.669 for the TCGA training and test datasets, respectively, showing a moderate predictive discrimination of the prognostic model. A nomogram was established, which showed a favourable predictive value for the prognosis of BLCA.
\end{abstract}

Conclusions: We developed and validated the performance of a prognostic model for BLCA that might facilitate the development of new biomarkers for the prognostic assessment of BLCA patients.

Keywords: Bladder cancer/BLCA, TCGA, RNA binding proteins, Prognostic model, Survival

\footnotetext{
* Correspondence: yfzhouwhu@163.com

${ }^{1}$ Hubei Cancer Clinical Study Center, Hubei Key Laboratory of Tumor

Biological Behaviors, Zhongnan Hospital, Wuhan University, Wuhan, China

${ }^{2}$ Department of Radiation Oncology and Medical Oncology, Zhongnan

Hospital, Wuhan University, Wuhan, China
}

(c) The Author(s). 2021 Open Access This article is licensed under a Creative Commons Attribution 4.0 International License, which permits use, sharing, adaptation, distribution and reproduction in any medium or format, as long as you give appropriate credit to the original author(s) and the source, provide a link to the Creative Commons licence, and indicate if changes were made. The images or other third party material in this article are included in the article's Creative Commons licence, unless indicated otherwise in a credit line to the material. If material is not included in the article's Creative Commons licence and your intended use is not permitted by statutory regulation or exceeds the permitted use, you will need to obtain permission directly from the copyright holder. To view a copy of this licence, visit http://creativecommons.org/licenses/by/4.0/ The Creative Commons Public Domain Dedication waiver (http://creativecommons.org/publicdomain/zero/1.0/) applies to the data made available in this article, unless otherwise stated in a credit line to the data. 


\section{Background}

Bladder cancer (BLCA) is the most common malignancy in the urinary system, ranking 4th among men and 18th among women [1]. The 5-year survival rates have remained generally flat since the 1990 s due to late diagnosis and limited therapeutics. Patients with 'nonmuscle-invasive' tumours are easier to treat and have lower mortality rate compared to those in patients with tumours that have grown into the muscle wall or beyond [2]. Currently, BLCA screening heavily relies on cystoscopy, upper urography, urine cytology, and computed tomography (CT) [3]. Cystoscopy is an invasive examination method that is also expensive and uncomfortable for patients. However, urine cytology is less sensitive. The measurement of circulating biomarkers is a promising diagnostic method owing to their relative availability in serum and plasma [2]. Thus, there is an urgent need to identify early diagnostic biomarkers and prognostic indexes to improve the treatment effects and survival rate of BLCA.

RNA-binding proteins (RBPs) interact with RNA to form ribonucleoprotein complexes that regulate RNA expression and function [4]. As important participants in post-transcriptional regulation, RBPs are involved in almost all post-transcriptional regulation processes, including RNA splicing, translation, transport, localisation, degradation, and stabilisation [5]. RBP dysregulation has been reported in multiple cancers, which affects tumorigenesis and development [5]. However, the knowledge of RBP-related mechanisms in the development of cancer remains rudimentary and inconclusive. Therefore, clarification of the roles of RBPs in BLCA will help us to better understand tumour pathogenesis and develop prognostic and response biomarkers.

Recently, various RBP-related mechanisms in cancer onset and progression have been clarified, including genomic alterations, transcriptional and post-transcriptional control, and posttranslational modifications [5]. In addition, RBPs directly or indirectly affected oncogenic and tumour-suppressive signalling pathways [6]. However, only a few RBPs have been completely studied and identified as vital players in human cancers. For example, PNO1, a novel RBP isolated from the human kidney, functions as an oncogene in urinary bladder cancer by promoting proliferation and inhibiting apoptosis of urinary bladder cancer cells [7]. ZFP36L1, a tandem zinc-finger RBP that mediates mRNA decay, acts as a tumour suppressor to regulate mRNAs involved in hypoxia and the cell cycle [8]. A recent study demonstrated that IMP3, a member of the insulin-like growth factor II messenger RNA binding protein (IMP) family, was significantly upregulated in muscle-invasive BLCA compared to non-muscular invasive tissues and could serve as an independent prognosis predictor for BLCA patients [9]. Previously, most research mainly focused on the correlation between a single or a limited number of RBPs and BLCA. A comprehensive study of RBPs functions will help us to fully understand their roles in BLCA. Therefore, this study downloaded RNA sequence data and corresponding clinical information concerning BLCA from The Cancer Genome Atlas (TCGA) database to screen for RBPs differentially expressed between tumour and normal samples. Subsequently, a series of bioinformatics analysis methods were performed based on these differential RBPs to finally identify eight independent prognosis-associated RBPs, which were then used to construct prognostic and nomogram survival models. The results of this study might facilitate the development of prognostic assessment models based on RBPs in patients with BLCA.

\section{Methods}

\section{Data processing}

We downloaded RNA sequence and corresponding clinical data from the TCGA database (TCGA, https://portal.gdc.cancer.gov/), including 19 normal samples and 414 BLCA samples. The negative binomial distribution method was used to identify differentially expressed RBPs between normal and BLCA samples [10]. The limma package (http://www. bioconductor.org/packages/release/bioc/html/limma. html) was used for analysis. Differentially expressed RBPs were screened using the criteria of false discovery rate $(\mathrm{FDR})<0.05$ and $\mid \log 2$ fold-change $(\mathrm{FC}) \mid>1$. The $\mathrm{R}$ package pheatmap (https://cran.r-project.org/ web/packages/pheatmap/index.html) was used to perform bidirectional hierarchical clustering of the expression values of the differentially expressed RBPs.

Gene ontology (GO) enrichment and Kyoto Encyclopaedia of genes and genomes (KEGG) pathway analyses

The biological functions of the differentially expressed RBPs were systematically examined by GO enrichment and KEGG pathway analyses using the $\mathrm{R}$ packages DOSE, clusterProfiler, enrichplot, ggplot2, etc. Both $P$ and FDR values $<0.05$ were considered statistically significant.

\section{Protein-protein interaction (PPI) network construction and module screening}

Differently expressed RBPs were submitted to the STRI NG database (http://www.string-db.org/) to detect PPIs [11]. The PPI network was then constructed and visualized using Cytoscape 3.7.0. The Molecular Complex Detection (MCODE) plug-in was used to screen the key modules from the PPI network with both MCODE scores and node counts $>5$ [12]. $P<0.05$ was considered statistically significant. 


\section{Prognostic model construction}

Eight independent prognosis-associated RBPs were identified by univariate and multivariate Cox regression. Afterward, the risk score model was constructed based on the expression levels and coefficients of the eight hub RBPs. The risk score of each BLCA patient was calculated using the following formula: Risk score $=\beta 1^{*} E x p 1+$ $\beta 2 * \operatorname{Exp} 2+\beta \mathrm{i}^{*} \operatorname{Exp} \mathrm{i}$, where $\beta$ represents the coefficient value of the independent prognosis-associated RBP, Exp represents the expression level of the independent prognosis-associated RBP, and $i$ represents $\mathrm{i}^{\text {th }}$ hub RBP.

\section{Validating the performance of the prognostic model}

The BLCA patients were divided into low- and high-risk groups according to the median risk score. Survival differences between the two groups were evaluated by the Kaplan-Meier method using log-rank tests. In addition, receiver operating characteristic (ROC) curves were used to determine the accuracy of the prognostic model [13]. Subsequently, calibration curves and the concordance index (C-index) were calculated using the rms (https:// cran.r-project.org/web/packages/rms/index.html) and the survcomp (http://www.bioconductor.org/packages/ release/bioc/html/survcomp.html) packages in R, respectively. A nomogram survival model was performed using the $\mathrm{R}$ package $r m s$ based on the eight independent prognosis-associated RBPs to predict the survival rate of BLCA patients at 1, 2, and 3 years. Univariable and multivariable Cox regression analyses were performed to assess the independent clinical prognostic factors in BLCA patients from TCGA.

\section{Verification of the prognostic value and expression levels} of the hub RBPs

The prognostic value of the eight RBPs in BLCA was assessed by plotting the Kaplan-Meier survival curves using log-rank tests. The Human Protein Atlas (HPA) online database (http://www.proteinatlas.org/) was used to investigate the differential expression of the eight hub RBPs at the protein level between tumour and normal tissues.

\section{Results}

Screening of differentially expressed RBPs

This study performed a series of bioinformatics techniques to comprehensively analyse the roles and

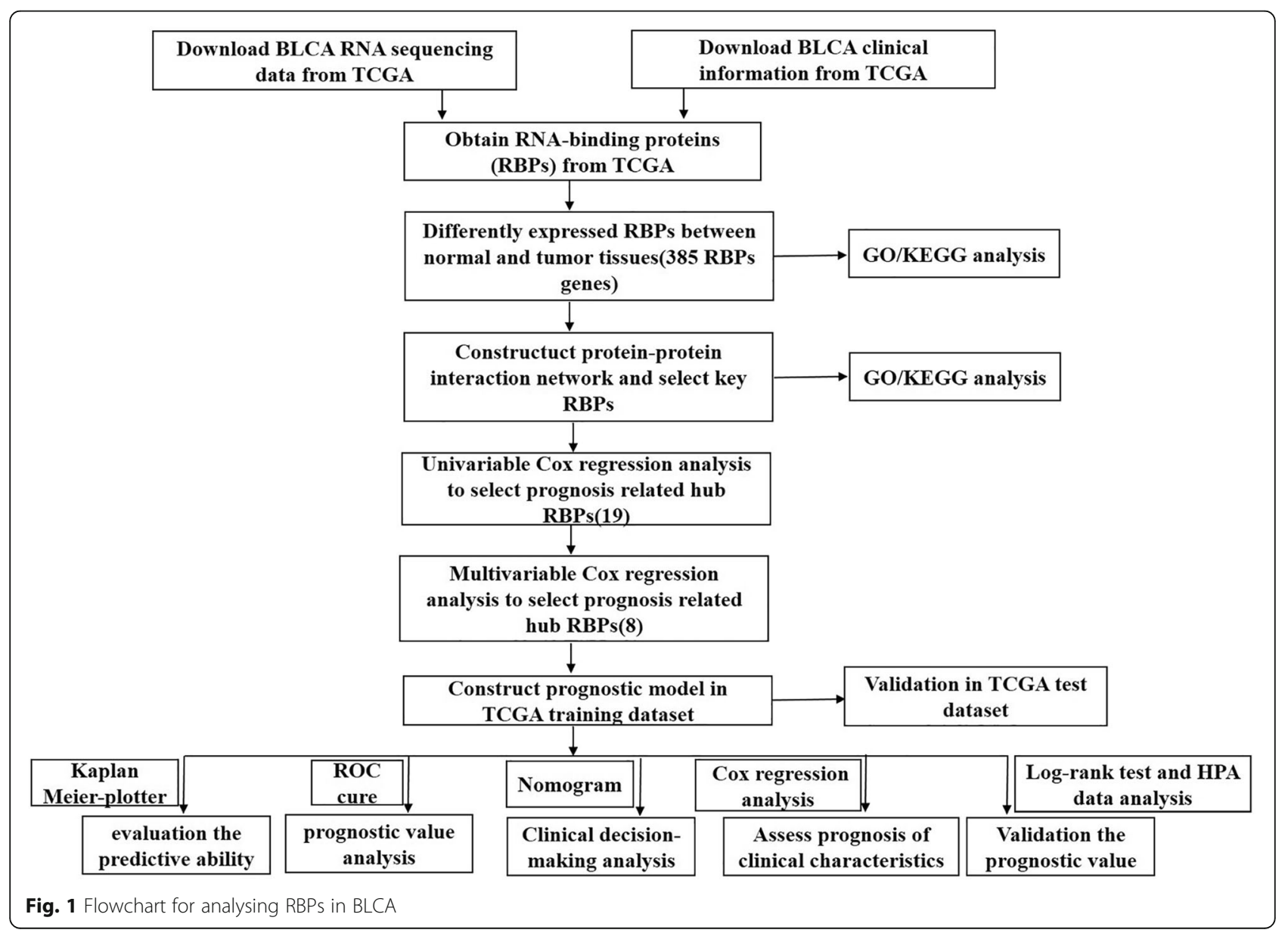


prognostic value of RBPs in BLCA. The flowchart of this study is shown in Fig. 1. We obtained RNA sequencing data and clinical information from the TCGA database containing 414 BLCA tissues and 19 normal tissues. The expression values of 1542 RBPs [4] were analysed in this study. A total of 385 differentially expressed RBPs were identified using the DEseq package that met the criteria of $P<0.05$ and $\mid \log 2 \mathrm{FC}) \mid>1.0$, including 218 upregulated and 167 down-regulated RBPs. The clustering heatmap and volcano plot of these differentially expressed RBPs are shown in Fig. 2.

\section{GO and KEGG pathway enrichment analysis of the differentially expressed RBPs}

To investigate the potential function and mechanisms of the identified RBPs, we divided these differentially expressed RBPs into upregulated and downregulated groups and performed GO and KEGG pathway enrichment analyses. The GO enrichment analysis showed that the biological processes of the upregulated RBPs were mainly enriched in ncRNA processing, tRNA metabolic processes, and RNA splicing, while the downregulated RBPs were mainly enriched for RNA splicing, regulation of cellular amide metabolic processes, and regulation of translation. The cellular component analysis indicated that the upregulated and downregulated RBPs were all primarily enriched in cytoplasmic ribonucleoprotein and ribonucleoprotein granules. The molecular function analysis showed that the upregulated RBPs largely enriched in catalytic activity, acting on RNA and ribonuclease activity; meanwhile, the downregulated RBPs were mainly enriched for translation factor activity, RNA binding, and mRNA 3'-UTR binding (Fig. $3 \mathrm{a}$ and b).
The KEGG pathway enrichment analysis showed that the upregulated RBPs were significantly enriched in aminoacyl-tRNA biosynthesis and cysteine and methionine metabolism, while the downregulated RBPs were enriched in lysine degradation and 2-oxocarboxylic acid metabolism (Fig. 3c and d).

\section{PPI network construction and key module screening}

To further explore the roles of differential RBPs in BLCA, Cytoscape was used to establish a PPI network comprising 373 nodes and 4063 edges based on the STRING database (Fig. 4a). Furthermore, the lines between the top 10 interacting proteins bolded according to the interaction scores. Subsequently, we used the MODE tool to analyse the co-expression network to identify the potential key modules. The most important modules comprised 104 nodes and 1151 edges (Fig. 4b). KEGG pathway analysis showed that the RBPs in these key modules were enriched for ribosome biogenesis in eukaryotes, spliceosomes, mRNA surveillance pathways, RNA polymerases, Huntington disease, cytosolic DNAsensing pathways, RNA transport, RNA degradation, ribosomes, and legionellosis.

\section{Identification of prognosis-related RBPs}

A total of 373 key differential RBPs were screened from the PPI network. To determine the association between RBPs and BLCA patients' outcomes, univariable Cox regression analysis was conducted to evaluate the prognostic value of these key differential RBPs which identified 19 hub RBPs (Fig. 5a). Subsequently, multivariable Cox regression analysis was performed to further analyse these 19 RPBs which showed eight hub RBPs to be $\mathbf{a}$

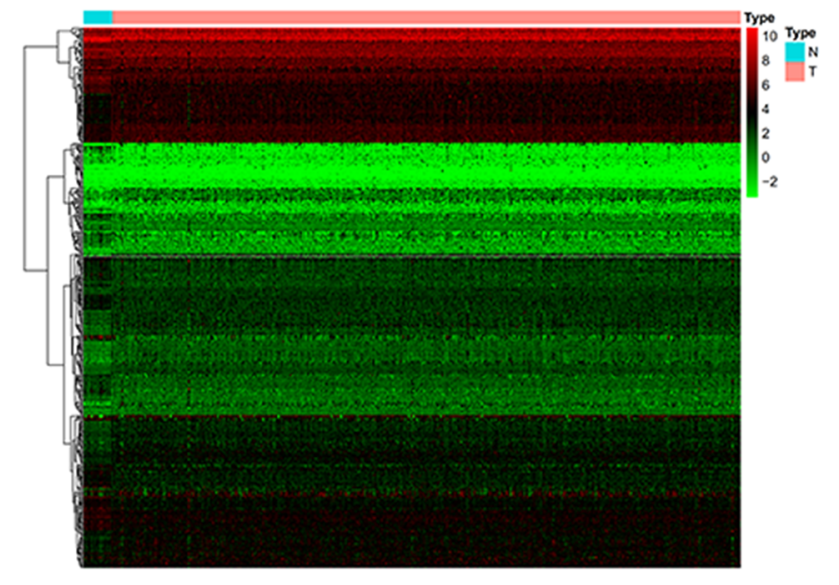

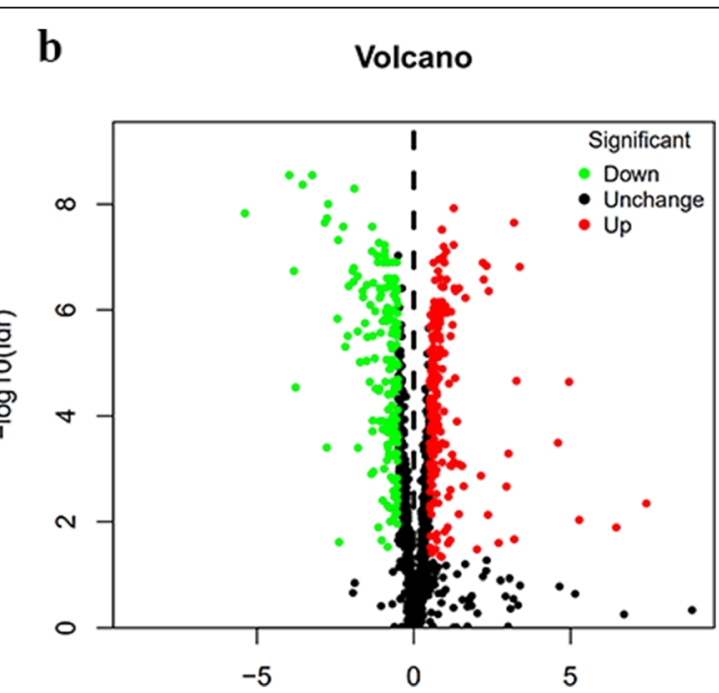

Fig. 2 Differentially expressed RBPs between BLCA and normal tissues. a Heatmap of differentially expressed RBPs. b Volcano map of the 1542 RBPs. The red, green, and black dots indicate high, low, and no difference in expression between bladder cancer and normal tissues 


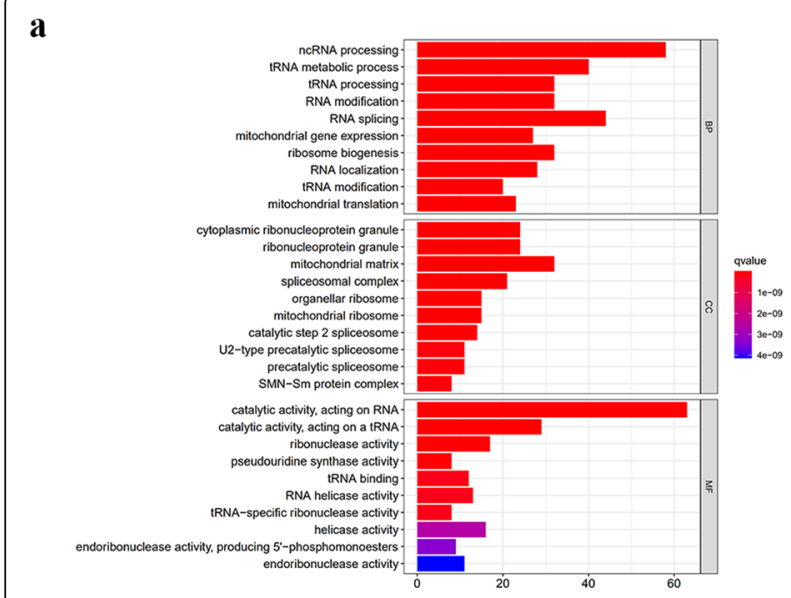

c

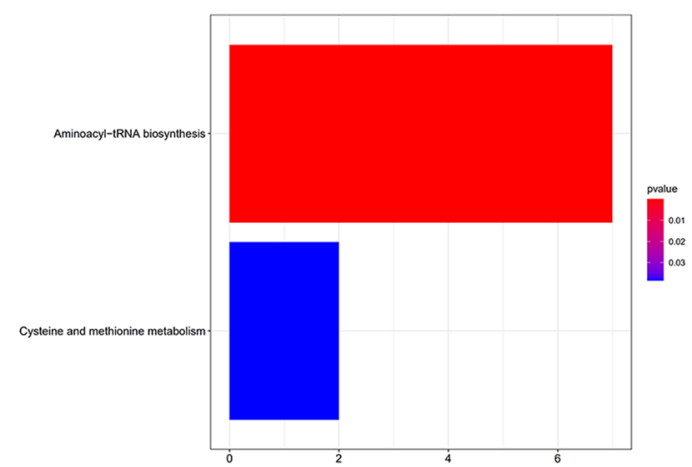

$\mathbf{b}$

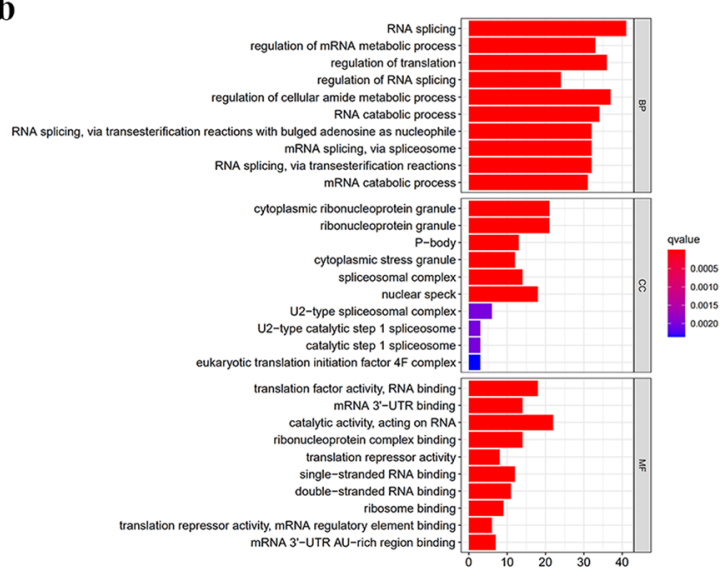

d

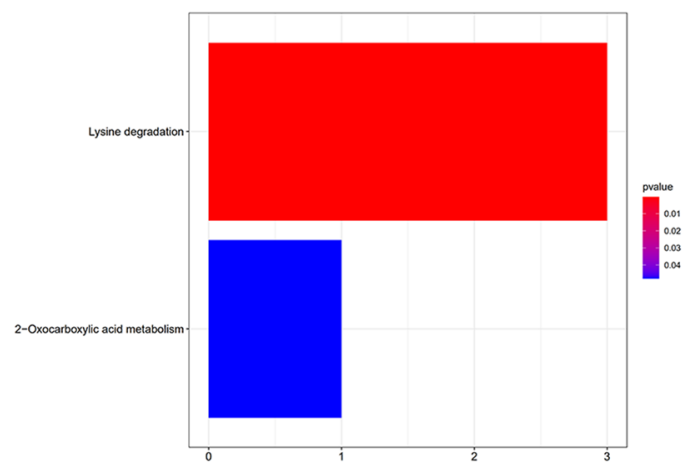

Fig. $3 \mathrm{GO}$ and KEGG pathway enrichment analysis of the differentially expressed RBPs. a GO enrichment analysis of upregulated expressed RBPs. b GO enrichment analysis of downregulated expressed RBPs. c KEGG pathway analysis of upregulated expressed RBPs. d KEGG pathway analysis of downregulated expressed RBPs

independent prognostic predictors in BLCA patients (Fig. 5b).

\section{Validation of the prognostic value and expression of hub} RBPs

To further investigate the prognostic value of these eight hub RBPs in BLCA, we plotted their Kaplan-
Meier survival curves to assess their relationships with overall survival (OS). Six hub RBPs (gem nuclear organelle associated protein 7 [GEMIN7], 2'-5'-oligoadenylate synthetase 1 [OAS1], apolipoprotein $\mathrm{B}$ mRNA editing enzyme catalytic subunit $3 \mathrm{H}$ [APO$B E C 3 H]$, aspartyl-tRNA synthetase 2, mitochondrial [DARS2], YTH domain containing 1 [YTHDC1], and a

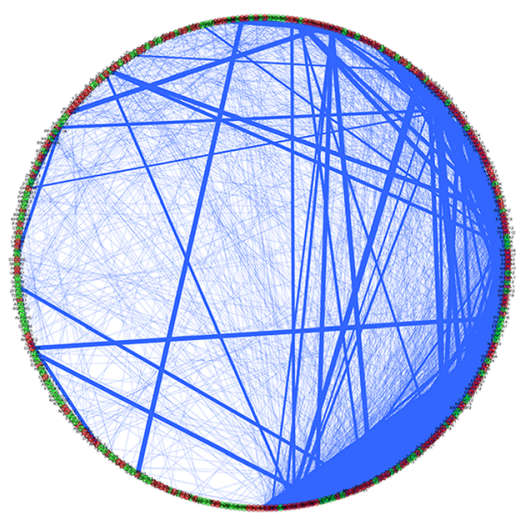

b

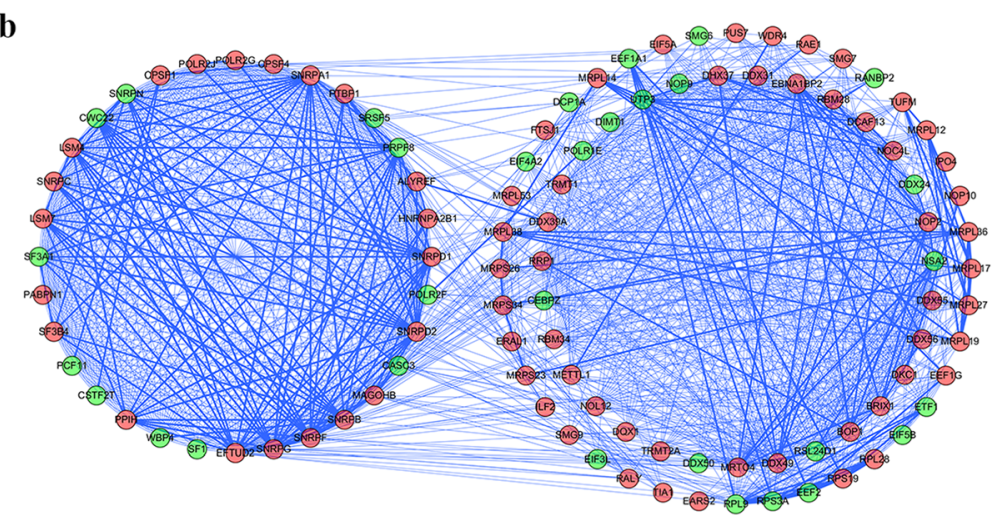

Fig. 4 PPI network and key modules analysis. a PPI network of differentially expressed RBPs. b Key modules from the PPI network. Green circles: down-regulated RBPs. Red circles: up-regulated RBPs. The bold lines represent the top 10 interacting proteins among these expressed RBPS 


\begin{tabular}{|c|c|c|}
\hline & pvalue & Hazard ratio \\
\hline EFTUD2 & 0.009 & $1.656(1.133-2.419)$ \\
\hline RPP21 & $<0.001$ & $0.623(0.473-0.821)$ \\
\hline GEMIN7 & 0.005 & $0.610(0.431-0.865)$ \\
\hline GARS & $<0.001$ & $1.498(1.185-1.894)$ \\
\hline XPO5 & 0.003 & $1.576(1.171-2.121)$ \\
\hline YARS & $<0.001$ & $1.787(1.288-2.480)$ \\
\hline OAS1 & $<0.001$ & $0.740(0.648-0.845)$ \\
\hline TIA1 & $<0.001$ & $0.616(0.492-0.773)$ \\
\hline АРОВЕС $3 \mathrm{H}$ & $<0.001$ & $0.642(0.496-0.831)$ \\
\hline TRIM71 & 0.008 & $1.683(1.144-2.474)$ \\
\hline DARS2 & 0.002 & $1.465(1.154-1.860)$ \\
\hline CLK2 & 0.006 & $0.666(0.499-0.891)$ \\
\hline YTHDC1 & 0.002 & $0.524(0.345-0.797)$ \\
\hline CLK1 & 0.007 & $0.743(0.600-0.921)$ \\
\hline MTG1 & 0.004 & $0.639(0.469-0.870)$ \\
\hline TYW3 & 0.001 & $1.564(1.198-2.041)$ \\
\hline PIN4 & 0.003 & $0.617(0.451-0.845)$ \\
\hline ZMAT3 & 0.009 & $1.306(1.068-1.598)$ \\
\hline RBMS3 & $<0.001$ & $1.713(1.259-2.331)$ \\
\hline
\end{tabular}
tein 3 [RBMS3]) were correlated with OS in BLCA patients (Fig. 6). Furthermore, we used immunohistochemistry results from the HPA database to further explore the protein expression levels of these hub RBPs in BLCA. The results showed higher tripartite motif containing 71 [TRIM71] expression in BLCA tissues compared to that in non-tumour tissues, b

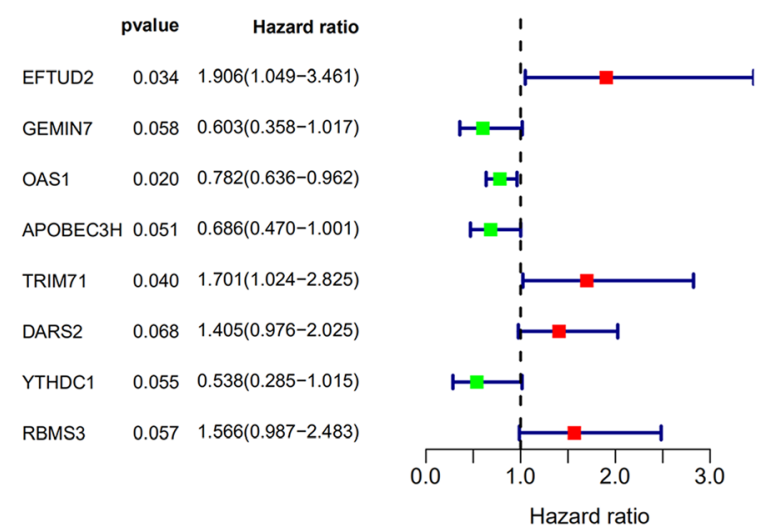

Fig. 5 Prognosis-related RBP selection by univariable and multivariable Cox regression analyses. a Univariable Cox regression analysis for the identification of prognosis-associated RBPs. b Multivariable Cox regression analysis for the identification of independent prognosis-related RBPs

a

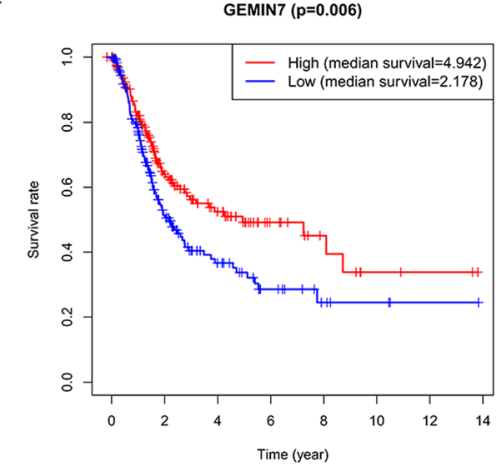

d

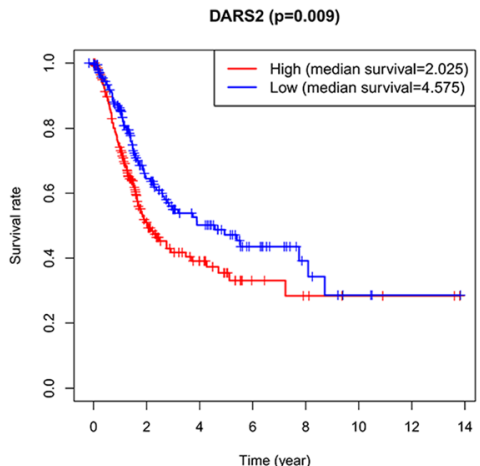

b

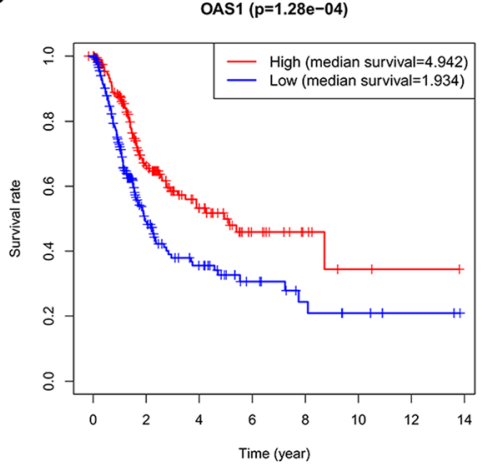

e

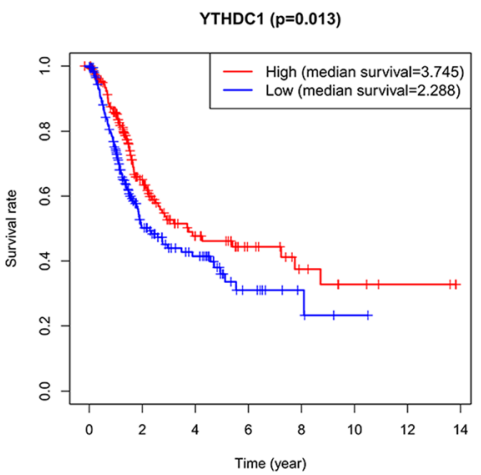

c

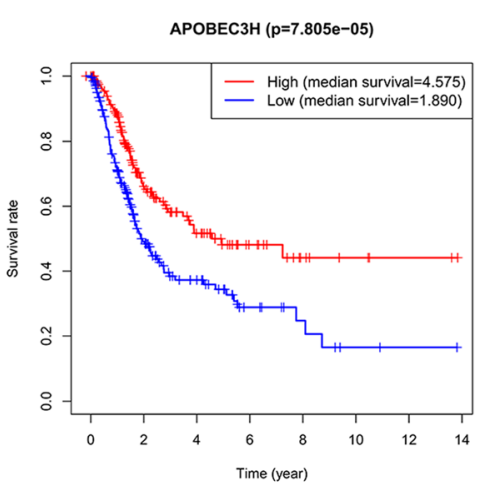

f

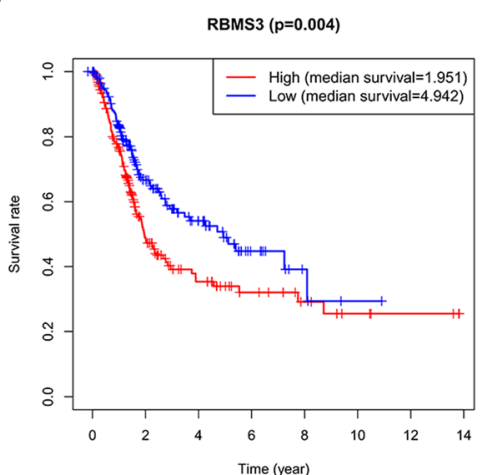

while DARS2 and RBMS3 expression levels were downregulated in tumour tissues. Meanwhile, there was no significant difference in the expression levels of OAS1, APOBEC3H, and YTHDC1 between tumour and normal tissues (Fig. 7). No data were available for elongation factor Tu GTP binding domain containing 2 [EFTUD2] and GEMIN7 in the HPA database.

Fig. 6 Survival analysis to verify the prognostic value of the hub RBPs in BLCA. Kaplan-Meier survival curves of a GEMIN7, b OAS1, c APOBEC3H, d DARS2, e YTHDC1, and $\mathbf{f}$ RBMS3. $P<0.05$ indicated statistical significance 


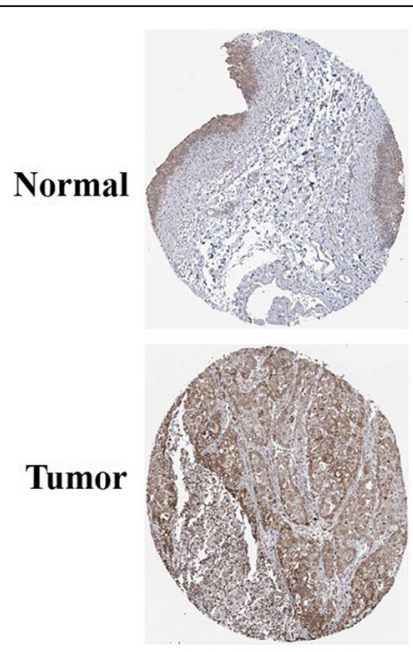

(a) TRIM71

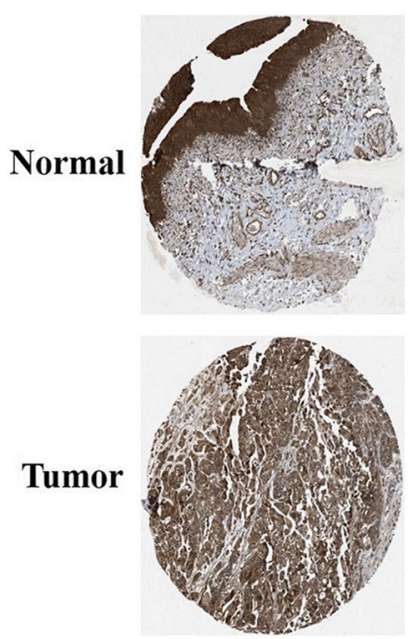

(d) OAS1

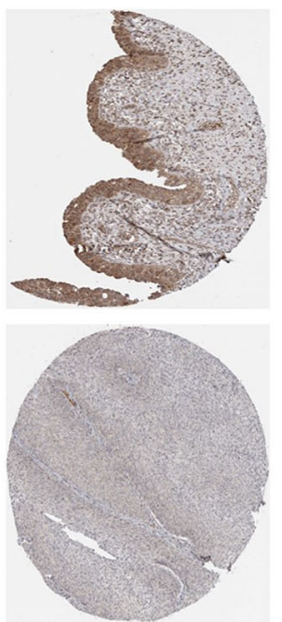

(b) DARS2
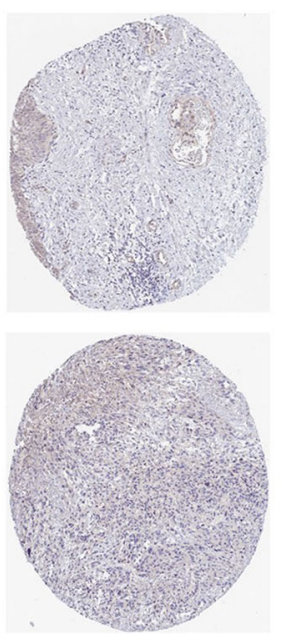

(e) АРОВЕС3Н

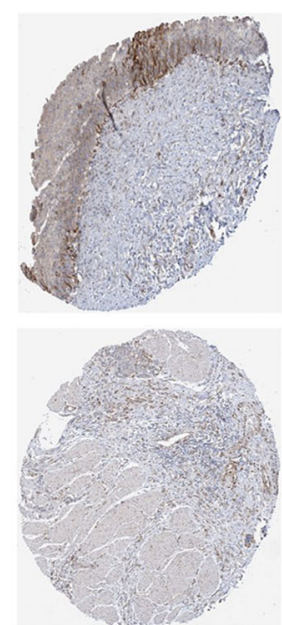

(c) RBMS3

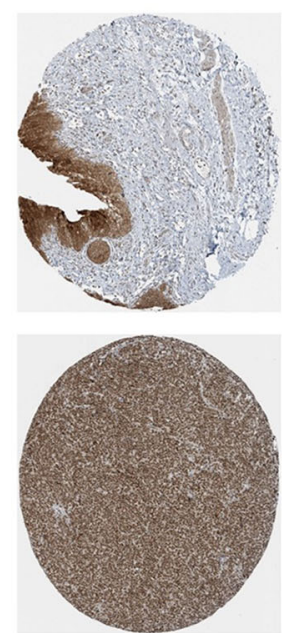

(f) YTHDC1

Fig. 7 Validation of hub RBP expression in BLCA and normal bladder tissue from the HPA database. Immunohistochemistry results of a TRIM71, $\mathbf{b}$ DARS2, c RBMS3, d OAS1, e APOBEC3H, and $\mathbf{f}$ YTHDC1

\section{Construction and analysis of a prognosis-related risk score model}

We established a prognosis-related risk score model based on the eight independent prognosis-associated RBPs. The risk score of each BLCA patient was calculated according to the following formula: Risk score $=(0.6449 *$ ExpEFTUD2 $)+(-0.5050 *$ ExpGEMIN7 $)+(-0.2456 *$ ExpOAS1 $)+(-0.3768 *$ ExpAPOBEC $3 \mathrm{H})+(0.5310 * \quad$ ExpTRIM71) $+(0.3403$ * ExpDARS2 $)+(-0.6204 *$ ExpYTHDC1 $)+(0.4484 *$ ExpRBMS3).

We then applied survival analysis to assess the predictive performance of this model. A total of 407 BLCA patients from TCGA were divided into the training and test datasets containing 204 cases and 203 cases, respectively. We then divided the 204 BLCA patients in the training dataset into low- and high-risk groups according to the median risk score. The result showed that patients in the high-risk group had a poor OS compared with those in the low-risk group (Fig. 8a). In addition, we constructed calibration plots and calculated the C-index, which was respectively $0.6368,0.6967$ and 0.6995 for OS prediction at the 1-, 2-and 3-year, suggesting a good conformity between the predicted and observed outcomes (Fig. 8b). Furthermore, a time-dependent ROC analysis [13], performed to further assess the prognostic power of the risk score model, showed an area under the ROC curve (AUC) of 0.795 (95\% confidence interval, 0.707-0.876) (Fig. 8c), indicating the favourable predictive discrimination of the prognostic model. Subsequently, BLCA patients in the training dataset were ranked by risk score to analyse their survival distribution. The heatmap showed the expression profile of the hub RBPs with increasing numbers of dead patients (Fig. 8d). 


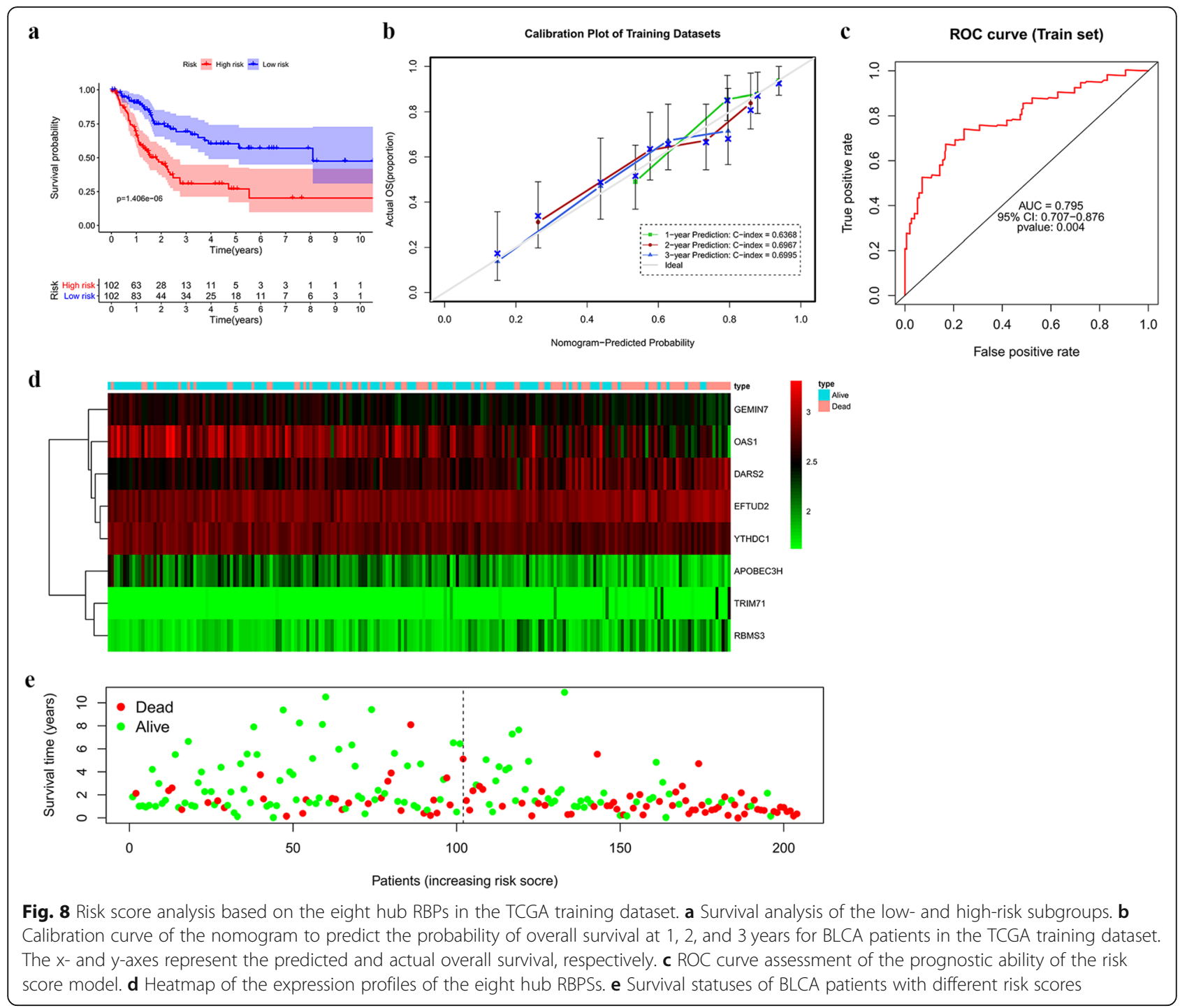

The scatter plot showed that the mortality rate of the patients increased with increasing risk score (Fig. 8e). We also validated the prognostic ability of this model in the test dataset, observing consistent results between the test and training datasets (Fig. 9a-e). These results showed acceptable sensitivity and specificity of the prognostic model.

\section{Construction of a nomogram}

To establish a quantitative prediction method for evaluating BLCA prognosis, we constructed a nomogram based on the eight independent prognosisassociated RBPs (Fig. 10). The point scale in the nomogram was used to assign points to each variable. We drew a vertical line to determine the points for each variable and summed the points of all variables to calculate the total points for each patient, which was then normalized to a distribution of 0 to 100 .
Hence, we could estimate the survival rates of BLCA patients at 1,2, and 3 years by calculating the total points for each patient by drawing a vertical line between the total point axis and each prognosis axis. This approach may help clinicians to make clinical decisions for BLCA patients.

Assessment of the prognostic value of clinical parameters To further assess the prognostic value of different clinical characteristics in BLCA patients from the TCGA database, we performed Cox regression analysis. The result of univariable analysis showed that age, tumour stage, and risk score were related to OS in BLCA patients (Fig. 11a). In the multivariable Cox regression analysis, only tumour stage and risk score remained independent prognostic indicators for BLCA patients (Fig. 11b). 


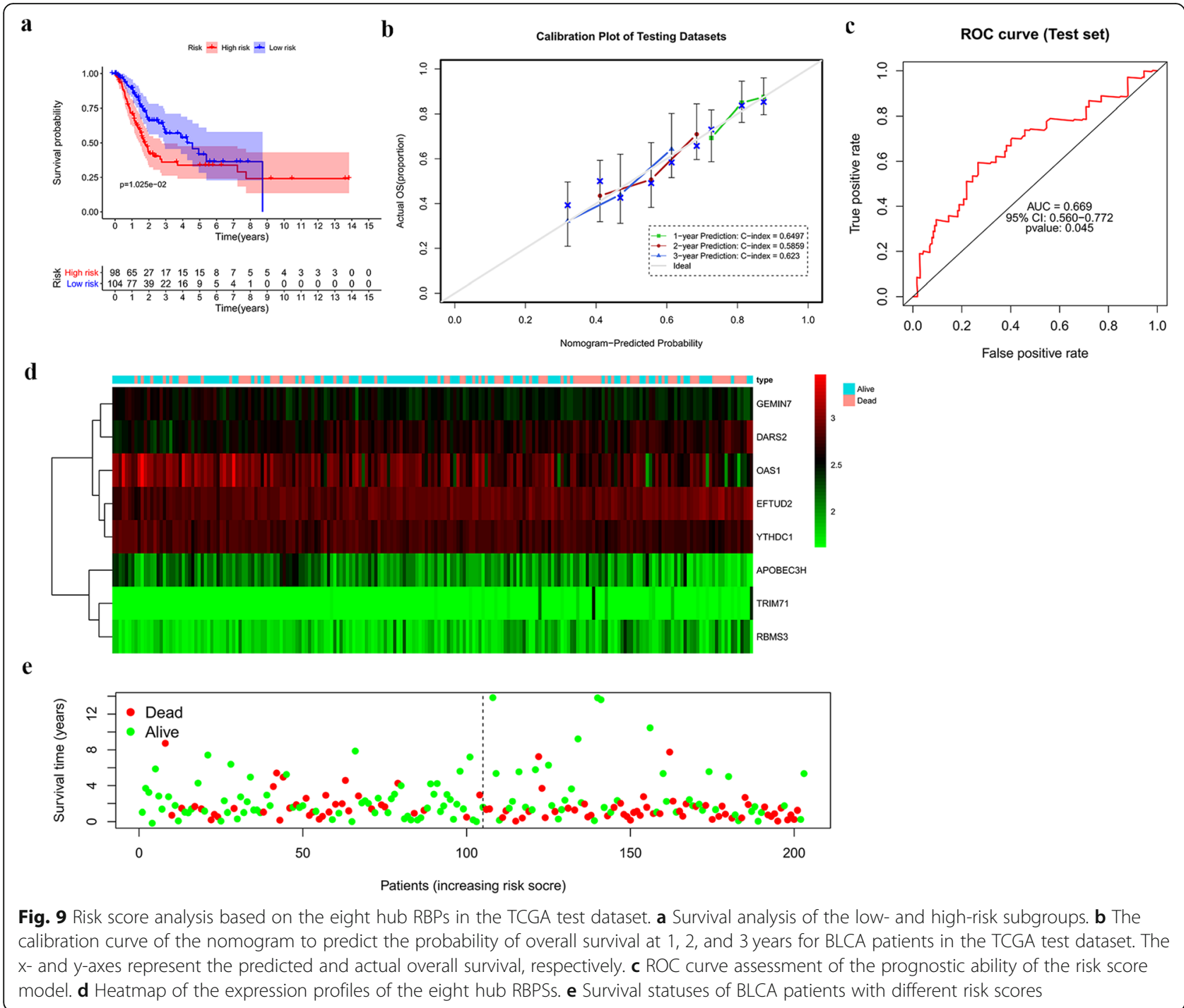

\section{Discussion}

Increasing evidence has confirmed the role of RBPs in carcinogenesis and some studies have consistently emphasized the association of RBPs as candidate biomarkers for patient prognosis and response to therapy in different cancer types [14-17]. However, how to apply these findings to clinical practice warrants further study. In the present study, we first screened 385 RBPs differentially expressed between BLCA and normal tissues from the TCGA database. Then, we systematically analysed the biological pathways and constructed PPI networks for these differential RBPs. Subsequently, we performed univariable and multivariable Cox regression analyses to further identify eight independent prognosisassociated RBPs. To further understand their biological functions and clinical significance, we also conducted survival and ROC analyses of the eight hub RBPs. Finally, we constructed a risk model based on these eight prognostic hub RBPs to predict the prognosis of BLCA patients. The results of our study provide new biomarkers for prognostic assessment of BLCA patients.

$\mathrm{GO}$ enrichment analysis showed that the biological processes (BPs) of the differently expressed RBPs were mainly enriched for ncRNA processing, tRNA metabolic processes, RNA splicing, regulation of mRNA metabolic processes, ribosome biogenesis, and translational regulation. Calo et al. reported that DExD-box helicase 21 (DDX21), a member of the DEAD-box RNA helicase family, was required for pre-rRNA processing; occupied the transcribed rDNA locus; directly contacted both rRNA and snoRNAs; and promoted rRNA transcription, processing, and modification in the nucleolus [18]. Protein quaking (QKI), a splicing factor frequently downregulated in lung cancer and correlated with poor prognosis, selectively suppressed the inclusion of NUMB mRNA exon 12 to promote 


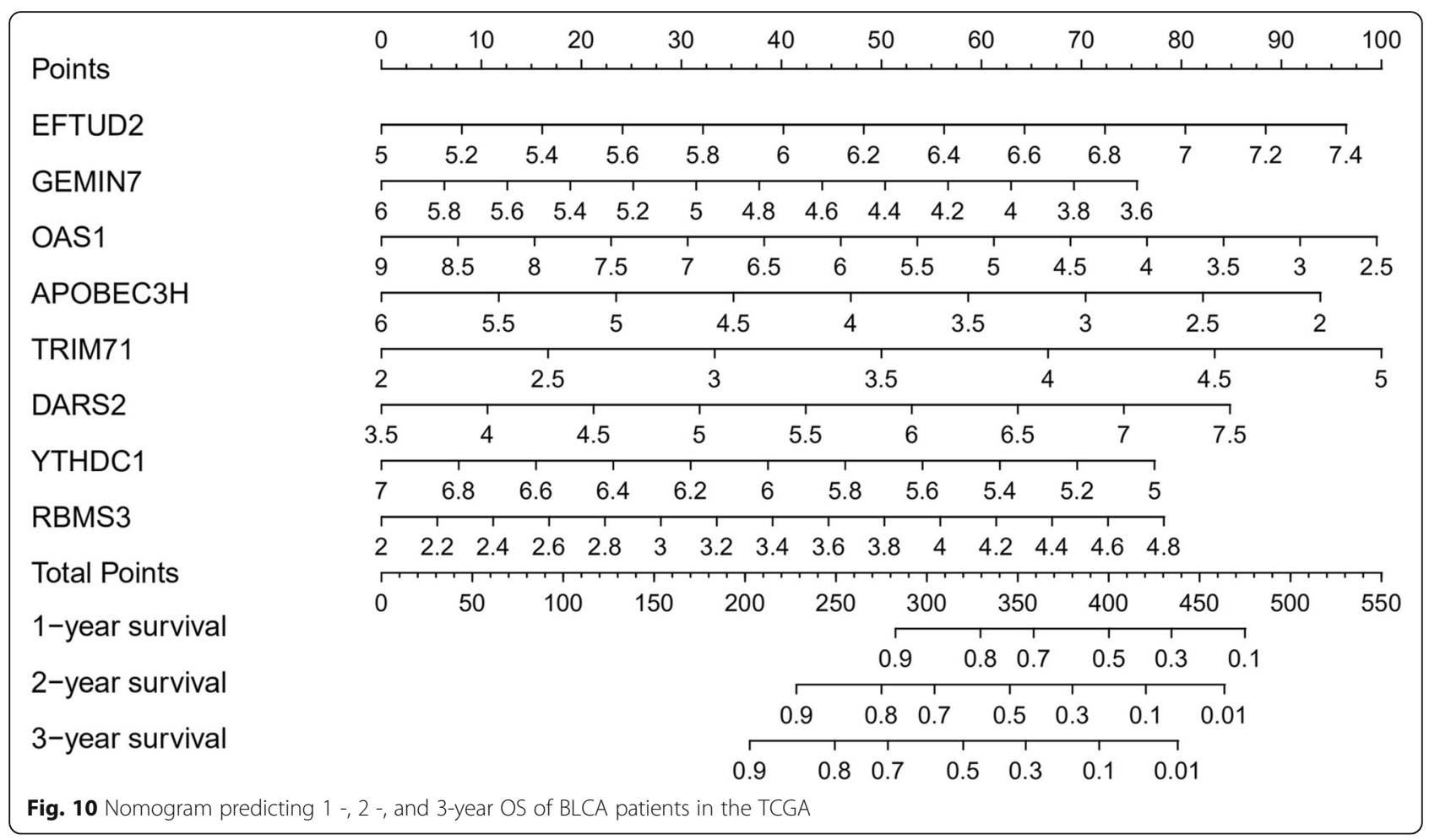

the expression of a NUMB isoform, thereby inhibiting proliferation and the Notch signalling pathway [19]. Another study reported that DEAH-box helicase 37 (DHX37), an ATP-dependent RNA helicase, was essential for ribosome biogenesis by facilitating small ribosomal subunit maturation. The cellular component analysis in the present study showed that the differential RBPs were primarily enriched for cytoplasmic ribonucleoprotein granule, ribonucleoprotein granule, mitochondrial ribosome, and P-body. Recently, Rozanska et al. demonstrated that ribosome binding factor A (RBFA) was a mitochondrial RBP that played important roles in mitoribosome biogenesis. RBFA combined with helices 44 and 45 of the $12 \mathrm{~S}$ rRNA in the mitoribosomal small subunit promoted the dimethylation of two highly conserved consecutive adenines, necessary for completing mitochondrial rRNA maturation and promoting the formation of a functional mitoribosome [20]. P-body, a cytoplasmic ribonucleoprotein granule, reportedly played a crucial role in translational repression and mRNA decay [21]. NBDY (NoBody), a recently identified P-body protein, inhibited mRNA turnover, as the silencing of NBDY expression destabilized a reporter

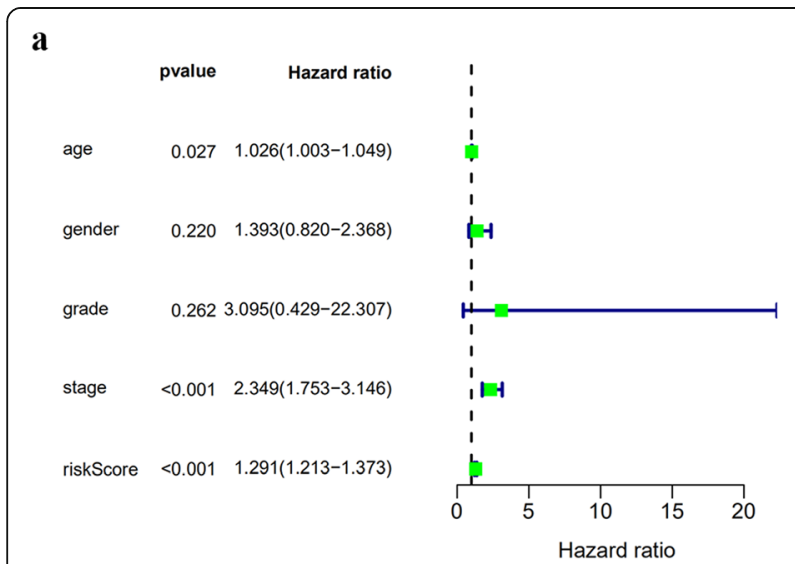

b

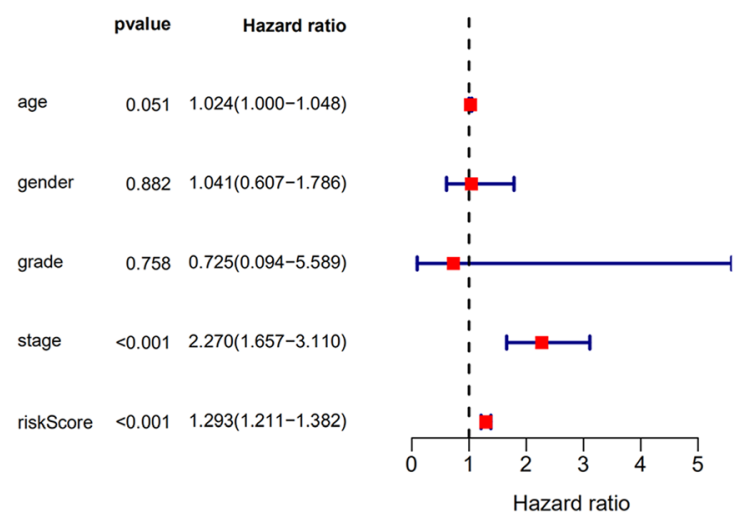

Fig. 11 Prognostic value of different clinical parameters. a Forest plots of univariable Cox regression analysis. b Forest plots of multivariable Cox regression analysis. $P<0.05$ indicated statistical significance 
of nonsense-mediated decay [22]. YTH N6methyladenosine RNA binding protein 2 (YTHDF2), another recently discovered P-body protein, is specifically bound to $\mathrm{N}$-methyladenosine and promoted the destabilization of mRNAs with this modification [23]. Regarding molecular function, the differential RBPs in the present study were largely enriched for catalytic activity, acting on RNA, ribonuclease activity, translation factor activity, and RNA and ribonucleoprotein complex binding. For example, mex-3 RNA binding family member $C$ (MEX-3C) is a MEX-3-homologous protein with E3 ubiquitin ligase activity mediated by a RING domain and critical for RNA degradation [24]. Cano et al. demonstrated that MEX-3C regulated HLA-A2 expression by binding to its 3 '-UTR, thereby inducing the ubiquitin-dependent degradation of this mRNA $[25,26]$.

The KEGG pathway analysis revealed that the differentially expressed RBPs were significantly enriched for aminoacyl-tRNA biosynthesis, methionine metabolism, lysine degradation, and 2-oxocarboxylic acid metabolism. A previous study reported that the faithful translation of genetic information from mRNA to protein is determined by two factors: the availability of aminoacyltRNAs composed of cognate amino acid-tRNAs pairs and the accurate selection of aminoacyl-tRNAs on the ribosome. Therefore, aminoacyl-tRNA biosynthesis, which is mediated by aminoacyl-tRNA synthetases, is crucial for translational quality control [27]. However, the role of RBP-mediated aminoacyl-tRNA biosynthesis in BLCA is unclear and warrants further study. AtGRP7, a known circadian clock regulated glycine-rich RBP, is an alternative splicing regulator [28]. Steffen et al. demonstrated that AtGRP7 loss-of-function mutants increased dimethylated lysine 4 levels in histone $\mathrm{H} 3$, which are markers of active transcription [29].

Subsequently, we established a PPI network of these differentially expressed RBPs and constructed a module containing 104 key RBPs. Most of these key RBPs have been reported to play important roles in cancer initiation, development, and metastasis. Cancer susceptibility candidate 3 (CASC3), also known as metastatic lymph node 51 (MLN51), is a splicing factor that regulates long intron-containing genes splicing. CASC3 overexpression significantly promoted hepatocellular carcinoma cell proliferation [30]. EFTUD2, an alternative splicing factor, may regulate the innate immune response in macrophages. Silencing of EFTUD2 expression significantly inhibited chronic intestinal inflammation and tumorigenesis, which was related to the reduced production of inflammatory cytokines and tumorigenic factors [31]. DDX39, a DEAD-box RNA helicase, was upregulated in hepatocellular carcinoma tissues and cells and negatively correlated with patient OS. Furthermore, DDX39 overexpression promoted hepatocellular carcinoma cell proliferation and invasion through the $\mathrm{Wnt} / \beta$-catenin pathway [32]. Block of proliferation 1 (BOP1), which reportedly participates in $28 \mathrm{~S}$ and $5.8 \mathrm{~S}$ ribosomal RNA processing and $60 \mathrm{~S}$ ribosome biogenesis, was downregulated in patient-derived melanoma samples [33, 34]. A loss of BOP1 also resulted in acquired resistance to BRAF kinase inhibitors in melanoma by increasing MAPK signalling [34]. Importin 4 (IPO4) belongs to the importin $\beta$ family, which is responsible for transporting histones $\mathrm{H} 3$ and $\mathrm{H} 4$ into the nucleus for chromatin assembly [35]. Xu et al. reported IPO4 overexpression in gastric cancer tissues and cell lines and demonstrated that IPO4 knockdown suppressed gastric cancer cell proliferation and migration [36]. This brief overview highlights the role of RBPs in tumorigenesis and development. Thus, the regulation of RBPs may represent an important breakthrough in tumour diagnosis, therapy, and prognostic prediction.

In this study, we finally identified eight independent prognosis-associated RBPs and used them to construct a prognostic prediction model. Moreover, the prognostic ability of this model was validated in the test dataset, which also showed a good predictive performance. Although five of the eight hub RBPs did not have statistically significant differences in the multivariate Cox regression analysis, these hub genes were found to be associated with OS in BLCA patients through survival analyses, which means these genes can be used to predict the prognosis of BLCA patients. Therefore, these five hub genes were also included when we constructed prognostic model. Among these hub RBPs, high expression levels of GEMIN7, OAS1, APOBEC3H, and YTHD $\mathrm{C} 1$ were associated with favourable prognosis in BLCA patients, while high expression levels of DARS2 and RBMS3 predicted poor prognosis. A previous study reported that GEMIN7, a component of the survival motor neuron complex, is involved in the biogenesis of the small nuclear ribonucleoprotein complex [37]; however, its role in cancers is rarely reported. OAS1, initially identified as an interferon-induced antiviral enzyme, was recently associated with 5-azacytidine (AZA) sensitivity, the deficiency of which resulted in the NCI-60 set of cancer cell lines resistant to AZA [38]. APOBEC3H is a single-stranded DNA cytosine deaminase that can induce mutations in tumour cells, resulting in immune recognition or cancer cell death [39]. YTHDC1, a $\mathrm{N}^{6}$ methyladenosine binding protein localized in YT-bodies adjacent to nuclear speckles, regulates mRNA splicing by recruiting splicing factors to the targeted mRNA [40]. DARS2 promoted cell cycle progression and inhibited hepatocellular carcinoma cell apoptosis via the miR-30e5p/MAPK/NFAT5 pathway [41]. Wu et al. demonstrated that the loss of RBMS3 in epithelial ovarian cancer not 
only induced chemoresistance to platinum but also promoted recurrence via miR-126-5p/ $\beta$-catenin/CBP signalling. Moreover, the loss of RBMS3 was associated with poor overall and relapse-free survival in epithelial ovarian cancer patients [42]. Another study found that RBMS3 inhibited breast cancer cell proliferation, migration, and invasion through the $\mathrm{Wnt} / \beta$-catenin signalling pathway [43]. The loss of EFTUD2 repressed colonic inflammation and tumorigenesis via inactivation of NF- $\mathrm{kB}$ signalling [31]. TRIM71, an E3-ubiquitin ligase, induced ubiquitination and degradation of mutant p53 by binding to its transactivation domain in ovarian cancer, leading to decreased ovarian cancer cell growth [44]. However, the functions and molecular mechanisms of these hub RBPs in BLCA remain poorly understood; thus, functional experiments are needed to further explore their potential roles and mechanisms. Moreover, large sample and multi-centre clinical studies are expected to verify the results.

In summary, our study systematically analysed the expression and prognostic value of differentially expressed RBPs in BLCA using a series of bioinformatics techniques. We finally identified eight independent prognosis-associated RSPs and successfully constructed a prognostic risk score model to effectively assess the prognosis of BLCA patients. To our knowledge, this is the first study to develop an RBP-related prognostic model for BLCA. This study provides a basis for the development of new therapeutic targets and prognostic biomarkers.

\section{Conclusion}

We developed a prognostic model for BLCA patients and validated the performance of the model, which might facilitate the development of new biomarkers for the prognostic assessment of BLCA patients.

\begin{abstract}
Abbreviations
APOBEC3H: Apolipoprotein B mRNA editing enzyme catalytic subunit $3 \mathrm{H}$; AUC: Area under the ROC curve; AZA: 5-azacytidine; BLCA: Bladder cancer; BOP1: Block of proliferation 1; BPs: Biological processes; CASC3: Cancer susceptibility candidate 3; CT: Computed tomography; DARS2: Aspartyl-tRNA synthetase 2, mitochondrial; DDX21: DExD-box helicase 21; DHX37: DEAHbox helicase 37; FC: Fold-change; FDR: False discovery rate; GEMIN7: Gem nuclear organelle associated protein 7; GO: Gene ontology; HPA: Human Protein Atlas; IMP: Insulin-like growth factor II messenger RNA binding protein; IPO4: Importin 4; KEGG: Kyoto Encyclopedia of Genes and Genomes; MEX-3C: Mex-3 RNA binding family member C; MCODE: Molecular Complex Detection; MLN51: Metastatic lymph node 51; NBDY: NoBody; OAS1: 2'-5'oligoadenylate synthetase 1; OS: Overall survival; PPI: Protein-protein interaction; QK1: Protein quaking; RBFA: Ribosome binding factor A; RBMS3: RNA-binding motif, single-stranded-interacting protein 3; RBPS: RNAbinding proteins; ROC: Receiver operator characteristic; TCGA: The Cancer Genome Atlas; TRIM71: Tripartite motif containing 71; YTHDC1: YTH domain containing 1; YTHDF2: YTH N6-methyladenosine RNA binding protein 2
\end{abstract}

\section{Acknowledgements}

This study was carried out using data from the TCGA. We are appreciative of the researchers who uploaded their data to the database. We are thankful to Editage (www.editage.cn) for English language editing.

\section{Authors' contributions}

C.F. and Z.Y. designed the study. C.F. drafted the manuscript. W.Q. revised the manuscript. C.F. and W.Q. conducted all data analyses. All authors read and approved the final manuscript.

\section{Funding}

This work was supported by the National Natural Science Foundation of China (81472799), the Health and Family Planning Commission of Wuhan Municipality (WX17Q38), and the Project of Hubei Medical Talents Training Program.

\section{Availability of data and materials}

The datasets used and/or analysed during the current study are available from the corresponding author on reasonable request. No additional permissions were required to use any of the repository data.

Ethics approval and consent to participate

Not applicable.

\section{Consent for publication}

Not applicable.

\section{Competing interests}

All authors state that there are no potential competing interests.

Received: 11 June 2020 Accepted: 17 February 2021

Published online: 08 March 2021

\section{References}

1. Siegel RL, Miller KD, Jemal A. Cancer statistics, 2020. CA Cancer J Clin. 2020; 70:7-30.

2. Grayson M. Bladder cancer. Nature. 2017:551:S33.

3. Ebrahimi H, Amini E, Pishgar F, Moghaddam SS, Nabavizadeh B, Rostamabadi Y, et al. Global, regional and national burden of bladder cancer, 1990 to 2016: results from the GBD study 2016. J Urol. 2019;201: 893-901.

4. Gerstberger $\mathrm{S}$, Hafner M, Tuschl T. A census of human RNA-binding proteins. Nat Rev Genet. 2014;15:829-45.

5. Pereira B, Billaud M, Almeida R. RNA-binding proteins in cancer: old players and new actors. Trends Cancer. 2017;3:506-28.

6. Ho JJD, Balukoff NC, Theodoridis PR, Wang M, Krieger JR, Schatz JH, et al. A network of RNA-binding proteins controls translation efficiency to activate anaerobic metabolism. Nat Commun. 2020;11:2677.

7. Lin C, Yuan H, Wang W, Zhu Z, Lu Y, Wang J, et al. Importance of PNO1 for growth and survival of urinary bladder carcinoma: role in core-regulatory circuitry. J Cell Mol Med. 2020;24:1504-15.

8. Loh XY, Sun OY, Ding LW, Mayakonda A, Venkatachalam N, Yeo MS, et al. RNA-binding protein ZFP36L1 suppresses hypoxia and cell-cycle signaling. Cancer Res. 2020;80:219-33.

9. Yang F, Zhou Q, Meng L, Xing N. IMP3 is a biomarker for non-muscleinvasive urothelial carcinoma of the bladder associated with an aggressive phenotype. Medicine. 2019;98:e16009.

10. Li W, Gao LN, Song PP, You CG. Development and validation of a RNA binding protein-associated prognostic model for lung adenocarcinoma. Aging. 2020;12:3558-73.

11. Szklarczyk D, Gable AL, Lyon D, Junge A, Wyder S, Huerta-Cepas J, et al. STR NG v11: protein-protein association networks with increased coverage, supporting functional discovery in genome-wide experimental datasets. Nucleic Acids Res. 2019;47:D607-3.

12. Bader GD, Hogue CW. An automated method for finding molecular complexes in large protein interaction networks. BMC Bioinformatics. 2003;4:2.

13. Heagerty PJ, Lumley T, Pepe MS. Time-dependent ROC curves for censored survival data and a diagnostic marker. Biometrics. 2000;56:337-44.

14. Sanchez-Jimenez C, Ludena MD, Izquierdo JM. T-cell intracellular antigens function as tumor suppressor genes. Cell Death Dis. 2015;6:e1669.

15. Molenaar JJ, Domingo-Fernandez R, Ebus ME, Lindner S, Koster J, Drabek K, et al. LIN28B induces neuroblastoma and enhances MYCN levels via let-7 suppression. Nat Genet. 2012;44:1199-206.

16. Kato T, Hayama S, Yamabuki T, Ishikawa N, Miyamoto M, Ito T, et al. Increased expression of insulin-like growth factor-II messenger RNA-binding 
protein 1 is associated with tumor progression in patients with lung cancer. Clin Cancer Res. 2007;13:434-42.

17. Abdelmohsen $\mathrm{K}$, Gorospe M. Posttranscriptional regulation of cancer traits by HuR. Wiley Interdiscip Rev RNA. 2010;1:214-29.

18. Calo E, Flynn RA, Martin L, Spitale RC, Chang HY, Wysocka J. RNA helicase DDX21 coordinates transcription and ribosomal RNA processing. Nature. 2015;518:249-53

19. Zong FY, Fu X, Wei WJ, Luo YG, Heiner M, Cao LJ, et al. The RNA-binding protein QKI suppresses cancer-associated aberrant splicing. PLoS Genet. 2014;10:e1004289.

20. Rozanska A, Richter-Dennerlein R, Rorbach J, Gao F, Lewis RJ, ChrzanowskaLightowlers ZM, et al. The human RNA-binding protein RBFA promotes the maturation of the mitochondrial ribosome. Biochem J. 2017:474:2145-58.

21. Hubstenberger A, Courel M, Benard M, Souquere S, Ernoult-Lange M, Chouaib R, et al. P-body purification reveals the condensation of repressed mRNA regulons. Mol Cell. 2017;68:144-e145.

22. D'Lima NG, Ma J, Winkler L, Chu Q, Loh KH, Corpuz EO, et al. A human microprotein that interacts with the mRNA decapping complex. Nat Chem Biol. 2017;13:174-80

23. Wang X, Lu Z, Gomez A, Hon GC, Yue Y, Han D, et al. N6-methyladenosinedependent regulation of messenger RNA stability. Nature. 2014;505:117-20.

24. Buchet-Poyau K, Courchet J, Le Hir H, Seraphin B, Scoazec JY, Duret L, et al. Identification and characterization of human Mex-3 proteins, a novel family of evolutionarily conserved RNA-binding proteins differentially localized to processing bodies. Nucleic Acids Res. 2007;35:1289-300.

25. Cano F, Bye H, Duncan LM, Buchet-Poyau K, Billaud M, Wills MR, et al. The RNA-binding E3 ubiquitin ligase MEX-3C links ubiquitination with MHC-I mRNA degradation. EMBO J. 2012;31:3596-606.

26. Cano F, Rapiteanu R, Sebastiaan Winkler G, Lehner PJ. A non-proteolytic role for ubiquitin in deadenylation of MHC-I mRNA by the RNA-binding E3ligase MEX-3C. Nat Commun. 2015;6:8670.

27. Ling J, Reynolds N, Ibba M. Aminoacyl-tRNA synthesis and translational quality control. Annu Rev Microbiol. 2009;63:61-78.

28. Heintzen C, Nater M, Apel K, Staiger D. AtGRP7, a nuclear RNA-binding protein as a component of a circadian-regulated negative feedback loop in Arabidopsis thaliana. Proc Natl Acad Sci U S A. 1997;94:8515-20,

29. Steffen A, Elgner M, Staiger D. Regulation of flowering time by the RNAbinding proteins AtGRP7 and AtGRP8. Plant Cell Physiol. 2019:60:2040-50.

30. Xu L, Dai W, Li J, He L, Wang F, Xia Y, et al. Methylation-regulated miR-124-1 suppresses tumorigenesis in hepatocellular carcinoma by targeting CASC3. Oncotarget. 2016;7:26027-41.

31. Lv Z, Wang Z, Luo L, Chen Y, Han G, Wang R, et al. Spliceosome protein Eftud2 promotes colitis-associated tumorigenesis by modulating inflammatory response of macrophage. Mucosal Immunol. 2019;12:1164-73.

32. Zhang T, Ma Z, Liu L, Sun J, Tang H, Zhang B, et al. DDX39 promotes hepatocellular carcinoma growth and metastasis through activating Wnt/ beta-catenin pathway. Cell Death Dis. 2018;9:675.

33. Strezoska Z, Pestov DG, Lau LF. Bop1 is a mouse WD40 repeat nucleolar protein involved in 28S and 5. 85 RRNA processing and 60S ribosome biogenesis. Mol Cell Biol. 2000;20:5516-28.

34. Gupta R, Bugide S, Wang B, Green MR, Johnson DB, Wajapeyee N. Loss of BOP1 confers resistance to BRAF kinase inhibitors in melanoma by activating MAP kinase pathway. Proc Natl Acad Sci U S A. 2019:116:4583-91.

35. Campos El, Fillingham J, Li G, Zheng H, Voigt P, Kuo WH, et al. The program for processing newly synthesized histones H3.1 and H4. Nat Struct Mol Biol. 2010;17:1343-51

36. Xu X, Zhang X, Xing H, Liu Z, Jia J, Jin C, et al. Importin-4 functions as a driving force in human primary gastric cancer. J Cell Biochem. 2019;120: 12638-s46.

37. Ogawa C, Usui K, Ito F, Itoh M, Hayashizaki Y, Suzuki H. Role of survival motor neuron complex components in small nuclear ribonucleoprotein assembly. J Biol Chem. 2009;284:14609-17.

38. Banerjee S, Gusho E, Gaughan C, Dong B, Gu X, Holvey-Bates E, et al. OASRNase $L$ innate immune pathway mediates the cytotoxicity of a DNAdemethylating drug. Proc Natl Acad Sci U S A. 2019;116:5071-6.

39. Hix MA, Wong L, Flath B, Chelico L, Cisneros GA. Single-nucleotide polymorphism of the DNA cytosine deaminase APOBEC3H haplotype I leads to enzyme destabilization and correlates with lung cancer. NAR Cancer. 2020;2:zcaa023

40. Xiao W, Adhikari S, Dahal U, Chen YS, Hao YJ, Sun BF, et al. Nuclear m(6)a reader YTHDC1 regulates mRNA splicing. Mol Cell. 2016;61:507-19.
41. Qin X, Li C, Guo T, Chen J, Wang HT, Wang YT, et al. Upregulation of DARS2 by HBV promotes hepatocarcinogenesis through the miR-30e-5p/MAPK NFAT5 pathway. J Exp Clin Cancer Res. 2017;36:148.

42. Wu G, Cao L, Zhu J, Tan Z, Tang M, Li Z, et al. Loss of RBMS3 confers platinum resistance in epithelial ovarian cancer via activation of miR-1265p/beta-catenin/CBP signaling. Clin Cancer Res. 2019;25:1022-35.

43. Yang Y, Quan L, Ling Y. RBMS3 inhibits the proliferation and metastasis of breast cancer cells. Oncol Res. 2018;26:9-15.

44. Chen Y, Hao Q, Wang J, Li J, Huang C, Zhang Y, et al. Ubiquitin ligase TRIM71 suppresses ovarian tumorigenesis by degrading mutant p53. Cell Death Dis. 2019;10:737.

\section{Publisher's Note}

Springer Nature remains neutral with regard to jurisdictional claims in published maps and institutional affiliations.
Ready to submit your research? Choose BMC and benefit from:

- fast, convenient online submission

- thorough peer review by experienced researchers in your field

- rapid publication on acceptance

- support for research data, including large and complex data types

- gold Open Access which fosters wider collaboration and increased citations

- maximum visibility for your research: over $100 \mathrm{M}$ website views per year

At BMC, research is always in progress.

Learn more biomedcentral.com/submissions 